\title{
Time in Classical and Relativistic Physics
}

\author{
Gordon Belot \\ Department of Philosophy \\ University of Michigan
}

Introduction

"Henceforth space by itself and time by itself are doomed to fade away into mere shadows, and only a kind of union of the two will preserve an independent reality"Minkowski inaugurated the modern, geometrical perspective on relativistic physics with this famous proclamation (Perrett and Jefferey, 1952, 75). Part of his point was that the notions of time and simultaneity play a very different role in the theory of special relativity than in classical physics. Of course, since Einstein arrived at the general theory of relativity by reflecting on how the physical principles underlying the special theory would need to be adapted in order to take account of gravity, it would be natural to guess that Minkowski's proclamation is at least as true of general relativity as of its parent theory.

What follows is a necessarily brief and incomplete survey of some of the ways that the notion of time functions in classical physics, in the theory of special relativity, and in the theory of general relativity. Perhaps surprisingly, it will emerge that while in many respects, the nature of time in general relativity is yet further removed from the nature of time in classical physics than it is in special relativity, there are also respects 
and circumstances relative to which general relativity supports temporal notions closer to those of classical physics than any that can be found in special relativity.

\section{Time in Classical Physics}

The notion of an instant of time plays an absolutely fundamental role in prerelativistic physics. Instants of time function in classical physics-and in commonsense - more or less in the way described by Newton:

we do not ascribe various durations to the different parts of space, but say that all endure simultaneously. The moment of duration is the same at Rome and at London, on earth and on the stars, and throughout the heavens ... as we understand any moment of duration to be diffused throughout all spaces, according to its kind, without any concept of its parts .... (Janiak 2004, 26)

Each instant of time extends everywhere-it is not like some places exist at some times, others at other times. And everything that happens, happens at some instant of time. As was pointed out already by Kant, it is helpful when thinking about the notion of simultaneity to think, not in terms of space and time, but in terms of a structure that unites them:

Accordingly, though time has only one dimension, yet the ubiquity of time, (to speak with Newton), in virtue of which all the things which can be thought sensitively are at some time, adds a further dimension to the magnitude of actual things, in so far as they hang, so to speak, from the same point of time. For, if you were to represent time by a straight line extended to infinity, and simultaneous things at any point of time by lines drawn perpendicular to it, the surface thus generated would represent the phenomenal world .... (Walford and Meerbote 1992, 394)

Today we call this encompassing structure spacetime. The spacetime of classical physics is a four-dimensional continuum. But it is often useful to pretend that space only has just 
two dimensions, so that we can visualize spacetime as a three-dimensional continuum.

For definiteness, let us picture the instants of time as forming horizontal planes, with spacetime consisting of a stack of such planes and later instants lying above earlier ones.

In classical physics, one generally assumes further that there is a natural measure of temporal duration: that for any two instants of time, there is a fact about the length of the temporal interval that separates them. ${ }^{1}$ So one normally takes the spacetime of classical physics to come equipped with some kind of gadget that encodes facts about the size of temporal intervals - e.g., a function that when given two instants of time returns the size of the interval of time that separates them. ${ }^{2}$

There is much more to say about the structure of spacetime in classical physics. Certainly, one will want to give the instants of time some geometrical structure (typically that of Euclidean geometry). And the most straightforward approaches to formulating laws of motion requires some further structure. Newton's own approach, featuring absolute space, can be thought of as involving the addition of a standard of rest-or, equivalently, of a notion of 'same place as'_picture a distinguished family of vertical lines, one through each spacetime point, each of which can be thought of as corresponding to a point of absolute space. ${ }^{3}$

\footnotetext{
${ }^{1}$ More carefully and plausibly: one assumes that for any four instants, there is a fact about the ratio of the size of the interval that separates the first pair to the size of the interval that separates the second pair. For the sake of clarity, I stick with the less cumbersome option in the text.

${ }^{2}$ Note though, that some standard approaches to formulating classical mechanics do not require such structures-see, e.g., Lanczos (1986, §§V.6 and V.7). Further, some masters of classical physics, such as Mach and Poincaré, denied that it made sense to think of there being standards for comparing sizes of intervals of time in abstraction from the detailed unfolding of physical events; see Mach (1960, §II.VI.2) and Poincaré (1958, Chapter II).

${ }^{3}$ Famously, there are other ways to proceed here. See, e.g., Stein (1967) or Earman (1992, Chapter 2).
} 
But for present purposes all we really need is the temporal structure of classical spacetime: spacetime falls into a family of instants of time, with each point of spacetime lying on exactly one such instant; and there is a natural measure of the interval of time that lies between any two instants.

Before moving on let us note a couple of conventions about how material events and objects are represented in classical physics and make a couple of further easy observations concerning time in classical physics.

We idealize events so that they occur at a point of space and an instant of time; we likewise idealize observers and other objects so that their temporal slices are single points. Events, then, are represented by single points in spacetime, while the history of an observer or other object is represented by a wordline - a curve in spacetime that crosses instants of time rather than lying within them. ${ }^{4}$ We can see, then, that no worldline ever forms a closed curve- for this would require doubling back and traveling 'backwards in time,' which is forbidden.

One last point. There is an interesting disanalogy between the notion of the temporal separation between events in spacetime and the notion of the distance between points in space (or between simultaneous events in spacetime). In the spatial case, we are sometimes interested in the distance from $A$ to $B$, sometimes in the distance from $A$ to $B$ along such and such path. That is, the notion of distance comes in two flavours: path-

\footnotetext{
${ }^{4}$ More precisely: worldlines are forbidden to ever be tangent to instants of time.
} 
independent and path-dependent. ${ }^{5}$ In the context of classical physics, there is no corresponding bifurcation of the of notion temporal separation: in classical physics there is no nontrivial path-dependent notion of duration - for any two events, there will be many worldlines that pass through both of them, but they will all agree that the amount of time that passes between the two events is just given by the extent of the duration that separates the instants upon which these events lie.

\section{Time in Special Relativity}

Famously, the situation is quite different in special relativity. Time travel is still forbidden. But one can no longer think of spacetime as falling into instants of time in a natural way. For some, but not all, pairs of events, there is a well-defined notion of the temporal separation between them. There is also a further, path-dependent, notion of temporal separation between events.

My goal in this section is to sketch the elegant, geometrical perspective on special relativity from which all of this follows. But it is probably worth pausing for a moment before plunging in, to review the foundational principles of the theory and a classic thought experiment that shows that these principles more or less mandate the abandonment any absolute notion of simultaneity.

We can think of Einstein's great achievement in the theory of special relativity as deriving from his willingness to cut the Gordian knot of difficulties that confronted the

\footnotetext{
${ }^{5}$ Of course, usually we can think of the former as a special case of the latter-in well-behaved spaces, the distance from $A$ to $B$ is typically the distance from $A$ to $B$ along the shortest route.
} 
electromagnetic worldview at the beginning of the twentieth century by: (i) taking absolutely seriously the principle of relativity, according to which the laws of physics do not distinguish between different observers undergoing inertial (i.e., force-free) motion; (ii) taking as axiomatic the light postulate, according to which the numerical value of the speed of light is the same when measured in any direction, by any inertial observer; and (iii) being willing to follow through to the end the consequences of (i) and (ii). ${ }^{6}$

Suppose that you stand midway between two telegraph poles by the side of some railway tracks. Your chum Cholmondeley sits perched atop a train speeding along those tracks, midway between the front of the locomotive and the back of the caboose. ${ }^{7}$ Around the time the train passes you, lightening strikes twice. One bolt of lightening strikes the front of the train - and the pole to your right, which the train is just pulling even with as lightening strikes. The other strikes the rear of the train — and the pole to your left, which the train is just pulling past as lightening strikes. You see the two lightening flashes at the same instant—and so you infer that the strikes were simultaneous (otherwise, since you stand midway between the poles, and since light travels the same speed in every direction, you would not have seen the flashes at the same moment). Cholmondeley of course, does not see the two flashes of light at the same time-after all, while both flashes are racing towards her, she is racing (much more slowly) towards the flash from

\footnotetext{
${ }^{6}$ The principle of relativity had, in one form or another, played a central role in physics since the $17^{\text {th }}$ century. The light postulate can be thought of as a mildly daring extrapolation from a family of experimental results of the late $19^{\text {th }}$ century, that showed, contrary to expectations, that it was impossible to detect the motion of the Earth relative to the ether through which light was supposed to propagate as sound propagates through air.

${ }^{7}$ You might want to look up the pronunciation of the name 'Cholmondeley,' if you have never come across it.
} 
the lightening bolt that struck the pole to your right and and away from the flash from the bolt that struck the pole to your left. So she sees the former flash just a bit earlier than the latter. But then she must think that the lightening strikes occurred at different times: after all, she sits midway between parts of the train that were struck - so, since light travels at the same speed in every direction, if the strikes had been simultaneous, she would have seen the flashes at the same time.

So far in this discussion we have leaned on the light postulate, and it has led us to a curious conclusion - that two observers can disagree about whether or not a given pair of events are simultaneous. But now let us invoke the relativity principle-nothing in nature allows us to privilege the account of one inertially moving observer over another. Applied to our case, this means that there is no question of who is right and who is wrong about the timing of the lightening strikes: relative to your state of motion, they were simultaneous; relative to Cholmondeley's they were not. Together, the light postulate and the principle of relativity (understood in the strong sense advocated by Einstein) more or less force us to give up on the idea that spacetime falls in a natural way into instants of time (since such a division into instants would give us an absolute notion of simultaneity that did not involve any relativization to observers or states of motion).

With that observation under our belts, let us turn to the geometry of Minkowski spacetime, the setting of the special theory of relativity. Let us continue to focus on the relatively easy to visualize case of a three-dimensional spacetime. Working in the style of analytic geometry, we identify Minkowski spacetime with the set of triples of real 
numbers, of the form $(t, x, y)$, equipped with the following gadget that encodes the spatiotemporal geometry:

For any points $A=\left(t_{1}, x_{1}, y_{1}\right)$ and $B=\left(t_{2}, x_{2}, y_{2}\right)$ the squared-interval between $A$ and $B$ is $d(A, B)=\left(t_{1}-t_{2}\right)^{2}-\left(x_{1}-x_{2}\right)^{2}-\left(y_{1}-y_{2}\right)^{2}$.

Notice that this looks an awful lot like the formula for calculating squares of distances in Euclidean space-except that the difference in the $t$-coordinates of $A$ and $B$ makes a positive contribution, while the differences along the $x$ and $y$ dimensions makes a negative contribution. It follows immediately that the squared-interval behaves quite differently from the square of the distance in Euclidean geometry: for any $A$, you can find a distinct point $B$ that will make $d(A, B)$ be positive, zero, or negative. Indeed, if you draw a picture, you will see that $d(A, B)=0$ if and only if the straight line connecting $A$ and $B$ makes a forty-five degree angle to the $t$-axis; it should then be easy to convince yourself that the squared-interval between $A$ and $B$ is positive if the straight line connecting them makes less than a forty-five degree angle with the $t$-axis - and that this quantity is negative if the line joining the points makes an angle of more than forty-five degree with the $t$-axis.

As one would expect, events are represented by points of Minkowski spacetime. It is a little trickier to single out the worldlines. Picture the $x$ - and $y$-axes of Minkowski spacetime as determining a horizontal plane, with the $t$-axis a vertical line. A curve in Minkowski spacetime counts as a worldline of a massive body if at each point on the curve, the tangent vector to that curve makes an angle of less than forty-five degrees to 
the $t$-axis - that is, a worldline of such a body is a curve that at each stage is tending upwards to a greater extent that in it tending towards any horizontal direction. ${ }^{8}$ The worldlines of massless bodies (such as photons) are those curves whose tangent vectors always make an angle of forty-five degrees to the $t$-axis - they always tend equally upwards and in a horizontal direction. ${ }^{9}$ Those wordlines that are also straight lines (i.e., consist of sets of points determined by a condition on coordinates like $a t+b x+c y=d$, for real numbers $a, b, c, d$ ) correspond to the histories of (massive or massless) bodies undergoing inertial motion.

If $A$ and $B$ are points in Minkowski spacetime with $d(A, B)>0$, then we say that the temporal separation between $A$ and $B$ is $t(A, B)=\sqrt{d(A, B)}$. When it is defined, $t(A, B)$ is a positive real number-but, of course, for many pairs of points of Minkowski spacetime, the temporal separation between them is simply not defined. We can think of $t(A, B)$ as the time that would pass between $A$ and $B$ as measured by an ideal clock carried by an inertial observer present at both events. Temporal separation behaves in some ways like distance in Euclidean geometry_but with differences deriving from the pesky minus signs that appear in the expression for the squared-interval. For instance, if $A, B$, and $C$ are events in Minkowski spacetime and $t(A, B)$ and $t(B, C)$ are both defined, then so is

\footnotetext{
${ }^{8}$ Recall that the direction of the tangent to a curve at a point is the direction of a line that 'just touches' the curve at that point.

${ }^{9}$ Forty-five degree angles play a special role here because we have chosen to work in units in which $c$, the speed of light, takes the value one. In more ordinary units, the formula for the squared-interval would have a factor of $c^{2}$ in front of its first term on the right hand side.
} 
$t(A, C)$ - and $t(A, C)$ is greater than or equal to $t(A, B)+t(B, C)$ (with equality occurring only on the special case where $A, B$, and $C$ lie on a straight worldline). ${ }^{10}$

In Minkowski spacetime, as in Euclidean geometry, it turns out to be interesting to introduce a path-dependent notion of the separation between two points. In either case, we can proceed as follows. We start with our expression for the separation between two points. We can think of this as giving us the length of the straight-line segment that joins these points. So we can assign a length to those jerky curves that connect our two points and which consist of a finite number of straight line segments strung together (with changes of direction at points where segments meet). This allows us to assign lengths to any curve - since any curve can be approximated arbitrarily well by jerky curves of this kind. In the setting of Minkowski spacetime, this gives us the means to define the following path-dependent temporal notion: if $A$ and $B$ are two points connected by a worldline of a massive body, then the length of that worldline is called the proper time associated with that worldline - it is to be thought of as the amount of time that would pass between $A$ and $B$ according to an ideal clock that traveled from $A$ to $B$ via the given worldline. A crucial feature of this notion is that for any worldline connecting $A$ to $B$, the proper time between $A$ and $B$ is less than or equal to $t(A, B)$, with equality only in the special case where the wordline is straight. In other words, among all observers present at

\footnotetext{
${ }^{10}$ Contrast this with the situation in Euclidean geometry, where the distance between points $A$ and $C$ is guaranteed to be less than the sum of the distance from $A$ to $B$ and the distance from $B$ to $C$-unless the three points are collinear, in which case we have equality.
} 
two events $A$ and $B$, the unique observer who ages the most between these events is the observer who moves inertially from $A$ to $B$.

The path-dependence of the notion of proper time and the special role played by inertial observers are often driven home with the following sort of story. Two twins are born-Daniel and Henrik. Soon thereafter, while Henrik floats freely in space, Daniel departs in a rocket ship, journeying hither and yon - until he eventually meets up with Henrik again. When Daniel returns he looks younger than Henrik: Daniel's wordline is one way to get from the twins' birth to their reunion, Henrik's is another; but because Henrik moves inertially in the interregnum, we know that more proper time passes along his worldline than along Daniel's. This parable is customarily called the 'twin paradox.' For consider the following line of thought, prima facie seductive to some:

It is a prediction of special relativity that observers see moving clocks as running slow. It should follow from this that each twin should expect the other to be the younger at their eventual reunion. And it should follow from the basic spirit of special relativity that either both must be right, or neither.

There are a number of confusions in this line of thought. For the present, let us just note that the version of the principle of relativity that is built into the special theory of relativity tells us that we must regard intertially moving observers as equivalent—but the only way to set up a twin-paradox-style story Minkowski spacetime is to have one twin accelerate at some point, which breaks any equivalence between the twins. ${ }^{11}$ This is enough to show that there is no paradox here. And indeed, from the geometrical perspective there is not even the threat of paradox: the geometry of Minkowski

\footnotetext{
${ }^{11}$ We will see below, though, that the situation is more complex in general relativity.
} 
spacetime tells us unequivocally that more proper time passes for an observer following an inertial trajectory between two events than for any other observer present at both events.

What about instants of time in special relativity? This is a somewhat complicated topic, in part because there are a number of things one might have in mind in speaking of instants of time in this setting. ${ }^{12}$ But I am going to keep things simple and ask: Can Minkowski spacetime can be thought of as composed of a stack of instants of time, each with endowed with Euclidean geometry, just as classical spacetimes can be?

In one sense, of course, the answer is yes: if you set $t=$ constant in our formula for the squared-interval between points in Minkowski spacetime, what you get (except for an annoying overall factor of -1 ) is the ordinary Euclidean rule for calculating (squares of) lengths in the horizontal plane picked out by the $t=$ constant condition. Put otherwise: each $t=$ constant plane in Minkowski spacetime has Euclidean geometry. So Minkowski spacetime certainly can be thought of as a stack of Euclidean planes - and it would of course be quite natural to think of these planes as instants of time-since e.g., no massless or massive body can travel between two points lying in a single such plane.

But in another more interesting and important sense Minkowski spacetime cannot be thought of as being composed of Euclidean instants in the same sense that classical spacetimes are. Think for a minute about how ordinary analytic geometry works. One introduces coordinate axes in the Euclidean plane, and an expression for the distance

\footnotetext{
${ }^{12}$ See Stein (1991) and the references therein.
} 
between any two points: $\sqrt{\left(x_{1}-x_{2}\right)^{2}+\left(y_{1}-y_{2}\right)^{2}}$. The use of coordinates makes many problems that were difficult in synthetic geometry more tractable. But of course, one also has to be a bit careful. In the synthetic, axiom-based, approach to Euclidean geometry, it is pretty clear that the Euclidean plane is homogeneous (no point is different from any other) and isotropic (no direction is different from any other). It may look like analytic geometry presents a different picture: once we set up our coordinate axes, there is a special point (the origin, where the axes intersect) and special directions (picked out by the axes). Learning to treat these features and their ilk as artifacts of the use of coordinates - rather than as subtle truths about Euclidean geometry that went unnoticed for many centuries - is part of coming to understand how to use coordinate-based methods to investigate Euclidean geometry.

Here it is helpful to appeal to the notion of symmetry. In the case of the Euclidean plane, we know before we start doing analytic geometry that translations and rotations of are symmetries of Euclidean geometry-transformations that preserve all geometric information. When we set up coordinate axes and start doing analytic geometry, it may look like we have forgotten this lesson, since rotations will not leave the coordinate axes fixed. But this is not so: analytic geometry does respect the rotation-invariance of Eucldiean geometry, but in a somewhat subtle way. For imagine rotating the $x$ - and $y$ axes that one starts with to obtain new $w$ - and $z$-axes. ${ }^{13}$ The Euclidean recipe for calculating squares of distances tells you to take the sum of the squares of the differences

\footnotetext{
${ }^{13}$ If one rotates through an angle $\theta$, then the $w-z$ coordinates of a point with $x-y$ coordinates $\left(x_{0}, y_{0}\right)$ will be $\left(x_{0} \cos \theta-y_{0} \sin \theta, x_{0} \sin \theta+x_{0} \cos \theta\right)$.
} 
between the two coordinates for each of two points - and it turns out that you always get the same answer, whether you work with the $w$ - and $z$-coordinates for a pair of points or with their $x$ - and $y$-coordinates. The fact that the formula for distance works just as well relative to either choice of axes tells us that, geometrically speaking, the $w-z$-coordinate system is just as good as the $x-y$-coordinate system—so any feature, such as special directions in the plane, that depends on the choice of coordinate system should be thought of as a mere artifact of the procedures of analytic geometry.

Something analogous happens in Minkowski spacetime. We can think of ourselves as having set up our coordinate-based version of Minkowksi spacetime by laying down certain coordinate axes and undertaking to use these coordinates and the formula for the squared-interval. But it turns out that there are other coordinate axes we could have used — coordinate axes that would look skew relative to our given ones if we drew a diagram. The recipe for calculating the temporal separation between a pair of points in Minkowski spacetime gives us exactly the same output whether we feed it the coordinates of these points relative to our original or our new coordinate system — which tells us that, from the point of view of spatiotemporal geometry, either set of coordinates is as good as the other. But if there is nothing special about the direction of our t-axis, then there is nothing special about the surfaces $t=$ constant—from the geometrical point of view, the surfaces $t^{\prime}=$ constant are just as good—and the two families of surfaces are skew with respect to one another. ${ }^{14}$

\footnotetext{
${ }^{14}$ In order to see how this works, consider the two-dimensional case and begin with coordinates $t$ and $x$. Consider a second set of coordinates related to the first as $t^{\prime}=\gamma(t-v x)$ and $x^{\prime}=\gamma(x-v t)$ where $v$ is a real
} 
Before moving on it is important to emphasize that these dry and dusty facts about coordinates have real physical implications (recall how you and Cholmondeley managed to disagree about which events were simultaneous). As Einstein observed, in the presence of the light postulate there is a natural rule that any inertially moving observer can use to determine which distant events are simultaneous: if a light signal sent out by you at time $t_{1}$ is reflected by event $E$ and returns to you at time $t_{2}$, then you will assume that event $E$ took place when the time shown by your clock was halfway between $t_{1}$ and $t_{2}$. Relative to this rule, for any pair of events you will (in principle) be able to say whether or not they are simultaneous with one another. The crucial point is that if you and another inertial observer each follow this procedure, then you will agree about which events are simultaneous if you are at rest with respect to one another-otherwise you will disagree, and use time axes that are skew with respect to one another.

\section{Time in General Relativity}

It is impossible here to give more than a few hints as to the workings of general relativity, Einstein's monumental theory that generalizes the special relativity in order accommodate gravity. General relativity is first and foremost a theory of the geometry of spacetime. To say that general relativity generalizes special relativity is to say that the geometry of Minkowski spacetime is one of the spatiotemporal geometries that is

number less than one and $\gamma=(1-v)^{-1 / 2}$. Confirm that the recipe for calculating the squared-interval gives the same value for any given pair of points, whichever set of coordinates one decides to employ. 
countenanced by general relativity: Minkowski geometry arises in the special case in which there is no matter or energy at all.

In addition to arising as a special case, Minkowksi spacetime also plays an absolutely central role in the theory: every general relativistic spacetime looks very similar to Minkowski spacetime if you look at small enough patches of it, just as the geometry of the surface of a sphere becomes harder and harder to distinguish from Euclidean geometry as one looks at smaller and smaller portions of it. ${ }^{15}$

Some general relativistic spacetimes look exactly like Minkowski spacetime at small scales. Obviously, Minkowski spacetime itself is one example. Other examples can be made by starting with Minkowski spacetime and 'cutting and pasting.' For example, we might: (i) start with two-dimensional Minkowski spacetime and choose inertial coordinates $x$ and $t$; (ii) throw away all points whose $x$ coordinate has an absolute value greater than one; (iii) decree that points of the form $(t, 1)$ and $(t,-1)$ are really the same point. That is: cut a strip out of Minkowski spacetime that is infinite in the $t$ direction and finite in the $x$ direction, then glue the edges of the strip together to form a spacetime that has the global structure of the surface of a cylinder. The resulting two-dimensional spacetime certainly does not look like Minkowski spacetime globally-space is now finite in extent. But each sufficiently small portion of this spacetime looks exactly like a portion of Minkowski spacetime.

\footnotetext{
${ }^{15}$ No portion of the sphere looks exactly like a portion of Euclidean space-e.g., in spherical geometry, the interior angles of a triangle always sum to greater than 180 degrees, and the ratio of the circumference of a circle to its diameter is always less than $\pi$. But these discrepancies get smaller and smaller as one looks at smaller and smaller figures on the sphere.
} 
Such examples are very special. In a typical general relativistic spacetime, the geometry is everywhere at least a bit different from that of Minkowski spacetime. In particular, typical general relativistic spacetimes have nonzero curvature - as opposed to Minkowski spacetime which is flat (i.e., has vanishing curvature). Curvature is a technical and nuanced notion. Roughly speaking: a (spatial or spatiotemporal) geometry is flat if, at least locally, it is possible to set up families of parallel lines that maintain their separation from one another; and a (spatial or spatiotemporal) geometry exhibits curvature if this is impossible - try as one might, one always finds that nearby lines diverge or converge. ${ }^{16}$ At the heart of general relativity lie equations that enforce a dependence of the geometry of spacetime on the distribution of matter and energy via a condition on spacetime curvature. ${ }^{17}$

In any general relativistic spacetime, curved or flat, at a point in spacetime the geometrical structure allows you to divide the curves passing through that point into three classes based on the directions picked out by their tangents: those that are suitable (at least at this one point) to represent the history of a massive object; those that are suitable (at least at this one point) to represent the history of a massless object; and others. Of course, a curve counts as the worldline of a massive object if it falls into the first category at each of its points.

\footnotetext{
${ }^{16}$ Choose two nearby points on the equator of a sphere: one might hope that the lines of longitude that run through those points would be parallel to one another; but not so-lines of longitude grow progressively closer to one another until they intersect at the poles.

${ }^{17}$ The dependence of geometry of the distribution of matter and energy means that a given spatiotemporal geometry will be consistent with only some ways that matter and energy might be distributed-e.g., Minkowksi geometry is permitted only in the vacuum case, in which spacetime is devoid of matter and energy. Further, the geometry of spacetime is not in general determined by the behaviour of matter and energy - e.g., the family of vacuum spacetimes includes all of the flat spacetimes, and many more besides.
} 
With this necessarily brief and less than fully satisfactory sketch in hand, we can turn to a quick consideration of the behaviour of time in general relativity.

Proper Time and Temporal Separation. In general relativity, as in special relativity, there is a rule for assigning lengths to curves. And for a worldline of a massive body, this length can again be interpreted as proper time: the time that would be measured by an ideal clock whose history is given by that worldline. As in special relativity, some worldlines correspond to bodies that are not subject to forces - in special relativity, these were inertially moving bodies, here they are freely falling bodies. ${ }^{18}$ If there is a curve that maximizes the proper time between two events, that curve is the worldline of a freely falling body. ${ }^{19}$

The notion of temporal separation is not quite so well-behaved. The obvious idea here would be to define the temporal separation between two points to be the maximum proper time that elapses between them according to any worldline that visits them both. This strategy works to perfection in well-behaved spacetimes. But in spacetimes that exhibit causal pathologies (our next topic) there are pairs of points, such that there is no upper bound on the length of curves that connect them. ${ }^{20}$

\footnotetext{
${ }^{18}$ In general relativity, gravity is absorbed into spacetime geometry rather than being treated as a forcethe effect of a body's gravitational field is mediated by the curvature of spacetime.

${ }^{19}$ Further, any worldline of a freely falling body locally maximizes proper time: if two points on such a curve are sufficiently close together, then no other curve sees as much proper time passing between them.

${ }^{20}$ Interestingly, there is a sense in which all of spacetime geometry is encoded in facts about proper timebut only if one restricts attention to causally well-behaved spacetimes.
} 
Time Travel. Consider what happens if we reverse the roles of $t$ and $x$ in the construction of cylindrical Minkowski spacetime - begin with a strip that is infinite in the $x$ direction but finite in the $t$ direction, then 'roll up' this strip by identifying opposite points along the edges. We again end up with a flat general relativistic spacetime. But now it is one in which time is finite and periodic while space is infinite. There are curves in this spacetime that are worldlines of freely-falling massive bodies and which are closed circles. $^{21}$ Each point along such a curve lies towards the future for a body following the curve - which is just to say that such body undergoes a continuous form of time travel, being sure at each point along its history that if it just waits long enough it will find itself in the immediate past of that point.

So general relativity permits a sort of time travel that is not permitted by the other theories we have considered. It is important to emphasize that this result is not an artifact of the sort of toy model we have been considering: there are a number of physically interesting general relativistic spacetimes that include closed worldlines - the most widely-discussed being the rotating worlds discovered by Gödel. Readers may be disappointed to hear, though, that the general relativistic spacetimes that best model the actual universe do not contain closed worldlines (and because of this fact, the wisdom of including time travel worlds in the content of general relativity has often been questioned).

\footnotetext{
${ }^{21}$ If you picture the spacetime as a cylinder with its axis running in a horizontal direction, then any crosssection of the cylinder that you get by intersecting it with a vertical plane orthogonal to this axis will be such a curve.
} 
Differential Aging in General Relativity. Consider again our original cylindrical Minkowski spacetime that is infinite in the temporal direction. Picture this spacetime as a cylinder with its axis pointing in the vertical direction.

If this cylinder had been made out of a strip of the Euclidean plane, it would have some obvious symmetries: rotating the cylinder about its axis or translating it along its axis would leave everything the same. Such a Euclidean cylinder obviously has privileged directions - the directions parallel or orthogonal to the axis. If we draw the family of lines along the surface of the cylinder that are parallel to the axis, this family is left invariant if we rotate the cylinder of if we translate along its axis. Similarly, if we draw the family of circles on the cylinder that arise if we intersect the cylinder with horizontal planes.

Exactly the same picture holds in spacetime case-our cylindrical Minkowski spacetime has the same symmetries (now interpreted as translation in time and rotation in space). And the same two families of curves on the cylinder are left invariant by these symmetries, whether we think of the cylinder as built out of a strip of the Euclidean plane or a strip of two-dimensional Minkowski spacetime. ${ }^{22}$

This is telling us that among all of the curves in cylindrical Minkowski spacetime that correspond to worldlines of freely falling bodies, there are some very special ones-

\footnotetext{
${ }^{22}$ Further, if we take into account certain reflection symmetries, then we find that no other families of curves are left invariant in this way.
} 
the ones that are given by straight lines parallel to the axis of the cylinder. Call these curves the worldlines of privileged observers.

If points $A$ and $B$ lie on the wordline of a privileged observer, then that observer sees more proper time pass between $A$ and $B$ then does any other observer associated with any other worldline that passes through $A$ and $B$. This may sound like something we are already very familiar with from special relativity. But here there is something new. For if $A$ and $B$ are the right distance apart along the worldline of the privileged observer, then there will be another freely falling observer who travels from $A$ to $B$ by following a curve that wraps one or more times around the cylinder between the two events.

This gives us a new twist on the twin paradox scenario. Apollo and Artemis are both subject to no forces, and each is present at both $A$ and $B$; Apollo is a privileged observer while Artemis is not; each sees the other as moving uniformly with respect to themselves. It follows from the geometry of spacetime that Apollo ages more between $A$ and $B$ than does Artemis - even though Artemis does not undergo acceleration at any point during her journey.

Let us have one more example in which there are again privileged observers $(=\mathrm{a}$ unique family of worldlines relative to which 'the geometry of space is constant in time'). Schwarzschild spacetime describes the geometry in the region external to an isolated, non-rotating massive body. For present purposes we can think about it very intuitively. We are entitled to the notion of observers at rest with respect to the central body. These are our privileged observers. Physically, of course, if you want stay at rest with respect to 
a massive body, you will need a rocket or some other means of accelerating — so in this setting, the privileged observers are not freely falling. Suppose that Peter is a privileged observer of this kind, maintaining a fixed distance $R$ from central body. And suppose that his brother Anton is a freely falling observer, executing circular orbits of radius $R$ about the central body. Then Peter and Anton will periodically encounter one another. Let $A$ be a point at which the worldlines of the two brothers coincide, and let $B$ be the very next one. Between $A$ and $B$, Anton circles the central body once while Peter stays at rest. Who ages more between $A$ and $B$-Peter who is a privileged observer, but accelerating, or Anton, who is not privileged, but is freely falling? Peter, as it turns out. This may seem to contradict what was said above - are we not guaranteed that the wordline (if any) that maximizes proper time between $A$ and $B$ should correspond to a freely falling observer? Indeed we are. Consider a third brother, Marian, who is also present at both $A$ and $B$ : at $A$ Marian is traveling directly outwards from the central body, with just enough initial velocity so that when he slows and begins to fall back inwards, he again meets Peter and Anton at $B$. Marian, it turns out, is the observer who maximizes proper time between $A$ and $B .^{23}$

Moral: neither being unaccelerated nor being a privileged observer is sufficient for maximizing proper time in general relativistic spacetimes.

\footnotetext{
${ }^{23}$ Physically, this is because Marian steals a march on Peter and Anton by moving away from the central body - in general relativity, clocks run slower the deeper they are in a gravitational field.
} 
SIMULTANEITY. There are many physically interesting general relativistic spacetimes that can be thought of as falling in a natural way into instants of time- that is, there are general relativistic spacetimes in which simultaneity is absolute (rather than relative, as it is in Minkowski spacetime). There are a variety of ways that this can happen. Here I mention just a few of the simpler scenarios.

(a) As we have seen, in some solutions there are privileged families of observers. In many spacetimes in which the privileged observers are freely falling, one finds that if each of the privileged observers carries out a version of the construction behind Einstein's simultaneity convention, then they end up agreeing with one another about the simultaneity relation.

(b) Some spacetimes have a high degree of symmetry and the symmetries single out a privileged family of instants of time-e.g., the versions of cylindrical Minkowski spacetime consider above.

(c) In spacetimes that have a beginning — as in the Big Bang models that are the workhorses of modern cosmology — there is, for each point in the spacetime, a worldline that stretches from the initial singularity to that point and which has maximum length among all such worldlines. So there is a privileged time function in such spacetimes that assigns to each point the proper time measured by the longest curve that connects that point to the initial singularity. We get often get a well-behaved notion of simultaneity by considering two points to be simultaneous just in case they are assigned the same value by this cosmological time function. 
Of course, it is not entirely clear what philosophers interested in time should make of this situation. After all, it is fairly certain that our own world does not correspond to any of the possibilities countenanced by general relativity (since our world, in contrast to general relativistic worlds, appears to be fundamentally quantum in nature). So one has to be cautious in drawing conclusions about the nature of time from facts about general relativity. Indeed, just because general relativity is a way station on the road to some future theory of quantum gravity, one might think that it more profitable to focus on structural features of its laws rather than on features of particular possibilities in attempting to understand the nature of time in our world. And with that rather daunting suggestion, I will bring this discussion to a close.

\section{Further Reading}

Original papers by Einstein, Minkowski, and others on special and general relativity are collected in Perrett and Jeffery (1952). For those interested in the fascinating history of those theories, a good starting point is Janssen and Lehner (2012).

There are many good nontechnical introductions to aspects of the special theory of relativity. I especially like: Chapter 3 of Salmon (1975), Chapter 2 of Maudlin (2011), and Mermin (2005). Giulini (2005) is a good choice for those ready, willing, and able to digest a more mathematical account.

Far and away the best introduction to conceptual apparatus of general relativity is Geroch (1978) - a masterpiece of honest but nontechnical exposition of physics. There are of course many textbook treatments of general relativity. To my taste, three of the best are (in order of increasing mathematical sophistication): Callahan (2000), Carroll 
(2004), and Wald (1984). For a thorough discussion of proper time and temporal separation in general relativistic spacetimes, see Beem, Ehrlich, and Easley (1996, Chapter 4).

Almost every good discussion of special relativity includes a discussion of the twin paradox. There is large and growing literature on the twin paradox in cylindrical Minkowski spacetime (and in related settings). Weeks (2001) and Luminet (2011) are helpful and include helpful bibliographies. For the twin paradox in Schwarzschild spacetime, see Grøn and Braeck (2011) and Sokołowski (2012).

There is a very large literature on time travel in general relativity. Gödel's papers on the topic are essential reading: these are reprinted with helpful editorial introductions in volumes two and three of Gödel's collected works—see Feferman et al. (1989) and Feferman et al. (1995). Rindler (2009) is a very helpful discussion of some of the physical ideas underlying Gödel's spacetime and related spacetimes and includes many useful references. Earman (1995) is the place to start for those interested in thinking about the philosophical implications of time travel in general relativity (and many other topics in the foundations of general relativity). Arntzenius and Maudlin (2002) is another classic discussion of some of this territory. For a recent overview, see Smeenk and Wütrich (2011). Maudlin (2007, 288-291) argues that general relativistic spacetimes that permit time travel should be viewed as being physically impossible, while Maudlin (2010) develops an interpretation of spatiotemporal geometry that takes proper time as basic (and which therefore presupposes that causally pathological spacetimes have been set aside). 
The literature on instants of time in special relativity is enormous. Interested readers should start with the classic Malament (1977) and the very helpful survey Giulini (2001); for Einstein's simultaneity convention in the general relativistic setting, see Sachs and $\mathrm{Wu}$ (1977) and O'Neill (1983) on proper time synchonizability. The literature on instants of time in general relativity (and with their connection with laws) is of course also very large. Those interested in this topic might start with Belot (2005) and (2006), and follow up on the references therein.

The viewpoint adopted here has been unapologetically geometric. There is some debate about whether this is really a good way to understand relativistic physics—see, e.g., Brown (2005) and Janssen (2009). 


\section{References}

Arntzenius, Frank and Tim Maudlin. 2002. "Time Travel and Modern Physics." In Time, Reality, and Experience, edited by Craig Callender, 169-200. Cambridge: Cambridge University Press.

Beem, John, Paul Ehrlich, and Kevin Easley. 1996. Global Lorentzian Geometry, $2^{\text {nd }}$ ed. Basel: Marcel Dekker.

Belot, Gordon. 2005. "Dust, Time, and Symmetry." British Journal for the Philosophy of Science 56: 255-291.

Belot, Gordon. 2006. "The Representation of Time and Change in Mechanics." In Philosophy of Physics, edited by Jeremy Butterfield and John Earman, 133-227. Amsterdam: North-Holland.

Brown, Harvey. 2005. Physical Relativity: Space-Time Structure from a Dynamical Perspective. Oxford: Oxford University Press.

Callahan, James. 2000. The Geometry of Spacetime: An Introduction to Special and General Relativity. New York: Springer-Verlag.

Carroll, Sean. 2004. Spacetime and Geometry: An Introduction to General Relativity. San Francisco: Addison Wesley.

Earman, John. 1992. World Enough and Space-Time: Absolute Versus Relational Theories of Space and Time. Cambridge MA: MIT Press.

Earman, John. 1995. Bangs, Crunches, Whimpers, and Shrieks: Singularities and Acausalities in Relativistic Spacetime. Oxford: Oxford University Press.

Feferman, Solomon, John Dawson, Stephen Kleene, Gregory Moore, Robert Solovay, and Jean van Heijenoort, eds. 1989. Kurt Gödel-Collected Works. Volume II. Oxford: Oxford University Press.

Feferman, Solomon, John Dawson, Warren Goldfarb, Charles Parsons, and Robert Solovay, eds. 1995. Kurt Gödel-Collected Works. Volume III. Oxford: Oxford University Press.

Geroch, Robert. 1978. General Relativity from A to B. Chicago: University of Chicago Press.

Giulini, Domenico. 2001. "Uniqueness of Simultaneity." British Journal for the Philosophy of Science 52: 651-670. 
Giulini, Domenico. 2005. Special Relativity: A First Encounter. Oxford: Oxford University Press.

Grøn, Øyvind And Simen Braeck. 2011. "The Twin Paradox in Cosmological Context." European Journal of Physics Plus 126: 79.

Janiak, Andrew, ed. 2004. Newton: Philosophical Writings. Cambridge: Cambridge University Press.

Janssen, Michel. 2009. "Drawing the Line Between Kinematics and Dynamics in Special Relativity." Studies in History and Philosophy of Modern Physics 40: 26-52.

Janssen, Michel and Christoph Lehner, eds. 2012. The Cambridge Companion to Einstein. Cambridge: Cambridge University Press.

Lanczos, Cornelius. 1986. The Variational Principles of Mechanics, $4^{\text {th }}$ ed. New York: Dover.

Luminet, Jean-Pierre. 2011. "Time, Topology, and the Twin Paradox." In The Oxford Handbook of Philosophy of Time, edited by Craig Callender, 528-5forty-five. Oxford: Oxford University Press.

Mach, Ernst. 1960. The Science of Mechanics. La Salle Illinois: Open Court.

Malament, David. 1977. "Causal Theories of Time and the Conventionality of Simultaneity." Noûs 11: 293-300.

Maudlin, Tim. 2007. The Metaphysics within Physics. Oxford: Oxford University Press.

Maudlin, Tim. 2010. "Time, Topology, and Physical Geometry." The Aristotelian Society-Supplementary Volume 84: 63-78.

Maudlin, Tim. 2011. Quantum Non-Locality and Relativity: Metaphysical Intimations of Modern Physics, $3^{\text {rd }}$ ed. Oxford: Blackwell.

Mermin, David. 2005. It's About Time: Understanding Einstein's Relativity. Princeton: Princeton University Press.

O'Neill, Barrett. 1983. Semi-Riemannian Geometry: With Applications to Relativity. San Diego: Academic Press.

Perrett, W. and G.B. Jeffery, trans. 1952. The Principle of Relativity: A Collection of Original Memoirs on the Special and General Theory of Relativity. New York: Dover.

Poincaré, Henri. 1958. The Value of Science. New York: Dover. 
Rindler, Wolfgang. 2009. "Gödel, Einstein, Mach, Gamow, and Lanczos: Gödel's Remarkable Excursion into Cosmology." American Journal of Physics 77: 498-510.

Sachs, Rainer and Hung-Hsi Wu. 1977. General Relativity for Mathematicians. Berlin: Springer-Verlag.

Salmon, W. 1975. Space, Time, and Motion: A Philosophical Introduction. Encino: Dickenson Publishing.

Smeenk, Chris and Christian Wütrich. 2011. "Time Travel and Time Machines." In The Oxford Handbook of Philosophy of Time, edited by Craig Callender, 577-630. Oxford: Oxford University Press.

Sokołowski, Leszek. 2012. "On the Twin Paradox in Statis Spacetimes: I. Schwarzschild Metric." Forthcoming in General Relativity and Gravitation.

Stein, Howard. 1967. "Newtonian Space-Time.” Texas Quarterly 10: 174-200.

Stein, Howard. 1991. "Relativity Theory and Openness of the Future." Philosophy of Science 58: 147-167.

Wald, Robert. 1984. General Relativity. Chicago: University of Chicago Press.

Walford, David and Ralf Meerbote, eds. 1992. Immanuel Kant: Theoretical Philosophy 1755-1770. Cambridge: Cambridge University Press.

Weeks, Jeffery. 2001. "The Twin Paradox in a Closed Universe." American Mathematical Monthly, 108: 585-590. 